\title{
Transcriptome sequencing of Pinus kesiya var. langbianensis and comparative analysis in the Pinus phylogeny
}

\author{
You-jie Zhao ${ }^{1,2+},{\text { Yong } \mathrm{Cao}^{2+}{ }^{+} \text {, Juan } \text { Wang }^{3} \text { and Zhi Xiong }}^{4^{*}}$
}

\begin{abstract}
Background: Pines are widely distributed in the Northern Hemisphere and have a long evolutionary history. The availability of transcriptome data has facilitated comparative transcriptomics for studying the evolutionary patterns associated with the different geographical distributions of species in the Pinus phylogeny.

Results: The transcriptome of Pinus kesiya var. langbianensis was sequenced using the Illumina HiSeq 2000 platform, and a total of 68,881 unigenes were assembled by Trinity. Transcriptome sequences of another 12 conifer species were downloaded from public databases. All of the pairwise orthologues were identified by comparative transcriptome analysis in 13 conifer species, from which the rate of diversification was calculated and a phylogenetic tree inferred. All of the fast-evolving positive selection sequences were identified, and some salt-, drought-, and abscisic acid-resistance genes were discovered.

Conclusions: $m R N A$ sequences of $P$. kesiya var. langbianensis were obtained by transcriptome sequencing, and a large number of simple sequence repeat and short nucleotide polymorphism loci were detected. These data can be used in molecular marker-assisted selected in pine breeding. Divergence times were estimated in the 13 conifer species using comparative transcriptomic analysis. A number of positive selection genes were found to be related to environmental factors. Salt- and abscisic acid-related genes exhibited different selection patterns between coastal and inland Pinus. Our findings help elucidate speciation patterns in the Pinus lineage.
\end{abstract}

Keywords: Pinus kesiya var. langbianensis, Transcriptome sequencing, Pinus phylogeny, Comparative transcriptomics

\section{Background}

Pines (Pinus) are widely distributed in the Northern Hemisphere and are the largest extant genus of conifers, constituting the most important source of wood in the forestry industry $[1,2]$. The 115 species of Pinus are divided into two subgenera [3] and possess a rich fossil record dating back as much as 130-140 million years ago (Mya) $[4,5]$. Many studies have focused on this genus, particularly with regards to its phylogenetic relationships [6, 7] and the timing of diversification events [6, 8-10]. However, no study has examined how pines adapted to their varied ecological environments over evolutionary history.

\footnotetext{
* Correspondence: 30085977@qq.com

†You-jie Zhao and Yong Cao contributed equally to this work.

${ }^{4}$ College of Light industry and Food, Southwest Forestry University, Kunming 650224, Yunnan, People's Republic of China

Full list of author information is available at the end of the article
}

Transcriptome sequencing technology can obtain all of the RNA information of an organism at a point in time, thereby providing a large amount of information for molecular biology studies [11-13]. In particular, Illumina SOLEXA sequencing has been widely used for the excavation and discovery of functional genes. Transcriptome sequencing can also obtain a large number of single nucleotide polymorphism (SNP), simple sequence repeat (SSR), and other molecular markers [14, 15]. Molecular markers with good polymorphism can help researchers shorten the genetic breeding cycle [16].

As increasing numbers of species have been included in transcriptome sequencing projects, comparative transcriptomics has received greater attention from researchers $[9,17-$ 19]. Comparative transcriptomics can elucidate the phylogenetic relationships of multiple species and can assess the functional differences between orthologous genes following divergence. The functional differences between orthologous

(c) The Author(s). 2018 Open Access This article is distributed under the terms of the Creative Commons Attribution 4.0 International License (http://creativecommons.org/licenses/by/4.0/), which permits unrestricted use, distribution, and 
genes constitute important evidence for studying patterns of evolution in different Pinus species from different geographical regions. Pinus species have adapted to different habitats, including low-latitude and high-latitude environments, and coastal and inland areas (Table 3). In Eurasia, P. sylvestris is mainly distributed in inland and high-latitude regions, whereas $P$. pinea, $P$. halepensis, and $P$. pinaster are coastal species distributed in the Mediterranean region and southern Europe. In North America, P. banksiana is distributed in the inland and high-latitude areas of North American and Canada, whereas P. taeda and P. palustris occur in the coastal areas of the southeastern United States. In Asia, $P$. tabuliformis is an inland and high-latitude Pinus species that occurs mainly in northern China.

Pinus kesiya var. langbianensis (P. kesiya var. langbianensis) is a coastal species in Asia, and it is mainly distributed in southwest China and southeast Asia. In this study, we performed transcriptome sequencing of $P$. kesiya var. langbianensis using the Illumina HiSeq2000 platform. Following sequence assembling and analysis, unigenes and some molecular markers in P. kesiya var. langbianensis were obtained. Comparative transcriptomics was subsequently used in 13 conifer species. A number of positive selection genes were determined to be related to environmental factors in the Pinus species from different geographical regions.

\section{Results}

\section{Transcriptome sequencing and de novo assembly}

The cDNA samples obtained from pine needles of $P$. kesiya var. langbianensis and other individuals were normalized to increase the sequencing efficiency of rare transcripts. A total of about 141 million reads with an average insert size of $200 \mathrm{bp}$ were generated from the Illumina HiSeq 2000 platform. After cleaning and quality control, about 133 million high-quality reads were obtained with a total length of $11.99 \mathrm{~Gb}$ (Table 1). Cleaned reads were de novo assembled to 68,881 unigenes with a mean size of $821 \mathrm{bp}$, representing a total length of about $42.5 \mathrm{Mb}$ and a mean size of $713 \mathrm{bp}$. More than half of the total assembly length of the unigenes was $>1402$ bp $(\mathrm{N} 50=1402)$. The coverage distribution revealed that most unigenes had a read-depth coverage of 20 -fold, and $29 \%$ unigenes were up to 100-fold (Additional file 1: Figure S1).
Functional annotation of the transcriptome

A total of 48,035 (69.74\%) P. kesiya var. langbianensis unigenes were significantly matched to known genes in the public databases (Table 2). Approximately 37,757 unigenes were aligned to the NCBI Non-redundant $(\mathrm{Nr})$ protein database. We examined the taxonomic distribution of the BLASTX best hits group by genus. As a result, only 8482 unigenes had specific functional annotations, $28.18 \%$ were within Picea, and $7.99 \%$ were within Pinus (Fig. 1). About 23,491 unigenes (34.10\%) were assigned to 42 functional groups based on Gene Ontology (GO) assignments (Additional file 2: Table S1), including biochemistry, growth, development, metabolism, apoptosis, and immune defense.

\section{Identification of SSRs and SNPs}

A total of 2382 distinct loci were identified, and the incidences of different repeat types were determined (Fig. 2). Among the different classes of SSRs, the tri-nucleotide repeats were the most abundant (40.22\%), followed by di-nucleotides $(28.76 \%)$. Similar results were found in other conifer species, with the exception of $P$. pinaster (Additional file 2: Table S2). More than 94,545 SNPs were identified from the $P$. kesiya var. langbianensis transcriptome (Fig. 3). Among all of the SNPs, transitions (61.61\%) were more frequent than transversions (38.39\%). A and G were the most frequent SNPs (31.20\%), and A and G were the second-most frequent (30.41\%).

\section{Orthologue identification and functional characterization of 13 conifer species}

Public transcriptome sequences were downloaded from the PlantGDB (http://www.plantgdb.org/) and NCBI databases (Table 3). The average number of unigenes was about 42,335 in the 13 conifer species, and P. taeda had the largest number of unigenes $(91,046)$, while $P$. tabuliformis had the least $(10,285)$. The average unigene length mostly exceeded $656 \mathrm{bp}$, except for $P$. sylvestris and P. monticola. All of the pairwise orthologues were identified by comparative analysis between the 13 conifer species (Table 4). The results showed that $P$. taeda had the maximum average number (7571) of orthologous genes, whereas $P$. sylvestris had the minimum average number (1552). The highest number of orthologous genes $(16,051)$ was found between $P$. kesiya var. langbianensis and P. halepensis, while the

Table 1 Transcriptome sequencing and assembly of $P$. kesiya var. langbianensis transcriptome

\begin{tabular}{lllc}
\hline Sequencing results & & Assembly results & \\
\hline Total number of raw reads & $141,234,042$ & Total number of unigenes & 68,881 \\
Total number of clean reads & $133,236,316$ & Total unigenes length (bp) & $56,537,842$ \\
Total clean Nucleotides (bp) & $11,991,268,440$ & Contig N50 (bp) & 1402 \\
Q20 (\%) & $98.25 \%$ & Mean length (bp) & 821 \\
N content(\%) & $0.01 \%$ & Max length (bp) & 15,498 \\
GC content (\%) & $45.02 \%$ & Min length (bp) & 200 \\
\hline
\end{tabular}


Table 2 Unigene annotation of $P$. kesiya var. langbianensis transcriptome

\begin{tabular}{llllllll}
\hline Unigenes & NR & NT & Swiss-Prot & KEGG & COG & GO & ALL \\
\hline 68,881 & 37,757 & 42,869 & 24,093 & 21,433 & 13,528 & 23,491 & 48,035 \\
\hline
\end{tabular}

lowest number (727) was found between $P$. sylvestris and $P$. halepensis. One hundred and thirty shared orthologues were found in all 13 conifer species (Fig. 4). The orthologues were annotated with GO terms (Additional file 2: Table S3), and 119 orthologues were involved in biological processes, cellular components, and molecular functions, and the other 11 orthologues had unknown biological functions.

\section{Phylogenetic and divergence time analysis}

The genetic distance of species is related to the synonymous mutation rate calculated by the orthologous genes, and thus the synonymous mutation rate was estimated for all of the pairs of orthologues in the 13 conifer species (Table 4). Pinus kesiya var. langbianensis and P. taeda yielded 13,493 orthologues with the synonymous substitutions per synonymous site (Ks) values exhibiting a normal distribution with a peak value of 0.04 . The minimum Ks peak was detected in $P$. kesiya var. langbianensis $(<0.01)$ with $P$. tabuliformis and P. sylvestris, followed by a $0.03 \mathrm{Ks}$ peak with $P$. banksiana and $P$. contorta, and a $0.08 \mathrm{Ks}$ peak with $P$. monticola and P. lambertiana, and the maximum Ks peak of 0.15 with the outgroup Picea glauca (Fig. 5). The relationship reflected by the Ks distance of $P$. kesiya var. langbianensis corroborated the Pinus phylogeny $[6,7]$. As observed in previous studies of six conifer species [9], $P$. tabuliformis had the same Ks peak of 0.03, 0.03, and 0.01 with $P$. taeda, P. contorta, and $P$. sylvestris, respectively.

Using Picea glauca as an outgroup species, the phylogenetic tree of Pinus was constructed based on the Ks peak distance matrix (Fig. 6). The phylogenetic relationships were highly consistent with the phylogenetic tree obtained from
cpDNA and mtDNA analyses in previous studies $[6,7]$. In the phylogenetic tree, the average Ks value was 0.146 between Pinus and Picea; 0.078 between subgenus Strobus and subgenus Pinus; and 0.035 between section Trifoliae and section Pinus. In the previous study of Picea sitchensis and P. taeda, it was calculated that the synonymous mutation rate of the molecular clock in genus Pinus was about $0.68^{*} 10^{-9}$ substitutions/site/year, and the divergence time could be expressed as $\mathrm{Ks}^{*} 10^{-6} / 0.68 * 10^{-9}$. Thus, the divergence time was estimated at about 214 Mya (Triassic) between Pinus and Picea; about 115 Mya (Cretaceous) between subgenus Strobus and subgenus Pinus; and about 51 Mya (Paleogene) between section Trifoliae and section Pinus.

\section{Evolutionary pattern of Pinus spp. genes}

The number of pairwise synonymous (Ks) and non-synonymous (Ka) substitutions per site can reflect the evolutionary patterns of species. $K a / K s>1$ indicates that the gene has been involved in positive selection during evolution (Table 5). A number of positive selection genes were found to be related to environmental factors (Table 6).

In section Pinus, 2750 positive selection genes were found between $P$. tabuliformis and $P$. kesiya var. langbianensis, including one salt stress-related gene $(\mathrm{Nr}$ annotation: NP_001105589.1) via the production of the NADP-dependent protein $[20,21]$. In section Trifoliae, $P$. taeda shared 1386 and 1685 positive selection genes with $P$. banksiana and $P$. contorta, including the same salt stress-related gene ( $\mathrm{Nr}$ annotation: NP_001105589.1). Furthermore, 1700 positive selection genes were identified between $P$. kesiya var. langbianensis and P. sylvestris, including a drought stress-related gene ( $\mathrm{Nr}$ annotation: AAD37375.1) via the production of the peroxidase protein [22, 23]. In section Quinquefoliae, 2688 positive selection genes were discovered between $P$. lambertiana and $P$. monticola, including one drought stress-related gene $(\mathrm{Nr}$ annotation: AAD37375.1). In genus Pinus, P. banksiana

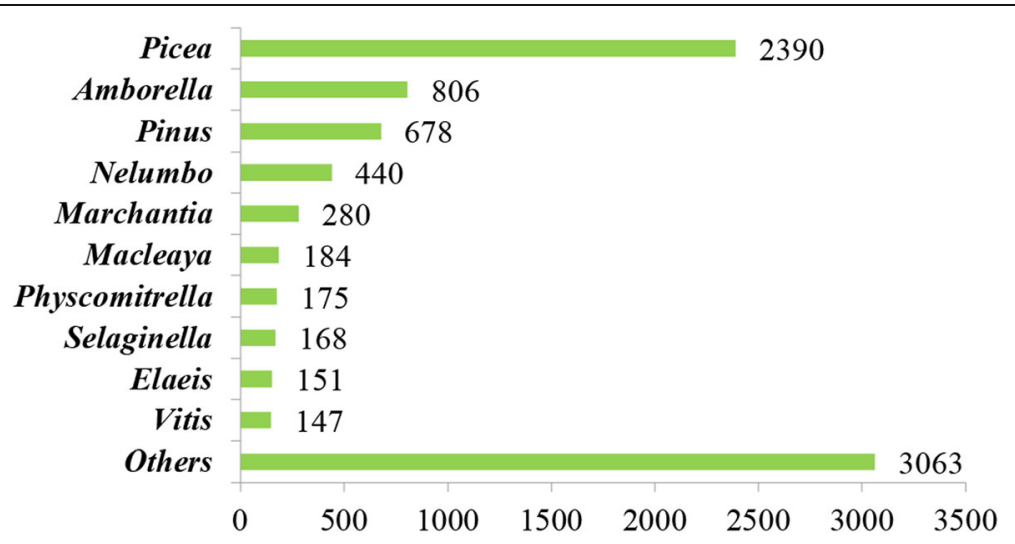

Fig. 1 Summary and taxonomic source of the BLASTX matches to unigenes. Number of unique best BLASTX matches of unigenes grouped by genus 


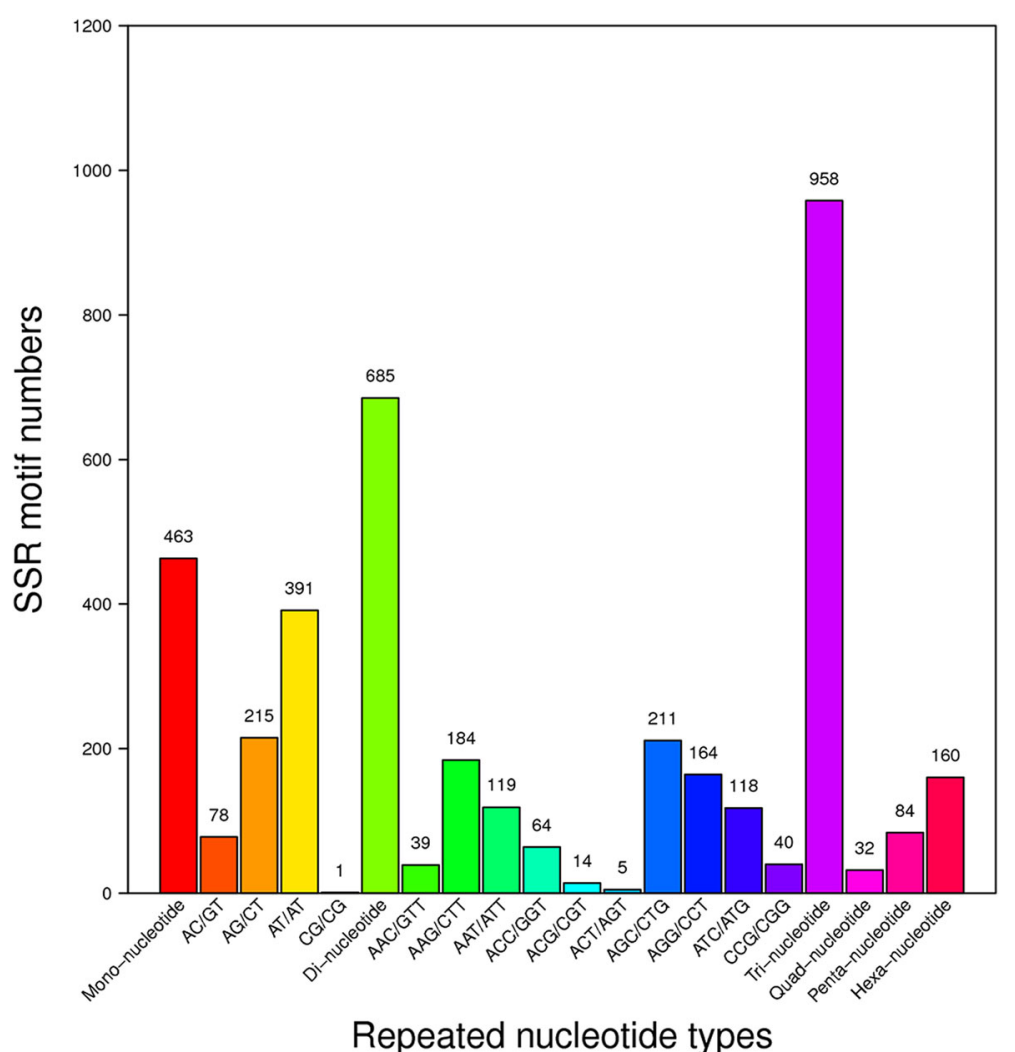

Fig. 2 Distribution of SSRs in the P. kesiya var. langbianensis transcriptome

shared 361, 603, 960, 1685, 1386, 1110, and 266 positive selection genes with $P$. pinea, $P$. halepensis, $P$. kesiya var. langbianensis, $P$. contorta, $P$. taeda, and P. palustris, and one gene related to abscisic acid stress ( $\mathrm{Nr}$ annotation: NP_001104929.1) was commonly found in all of the pairs.

The results indicate that salt and abscisic acid stress genes were obviously involved in positive selection between coastal and inland Pinus species.

\section{Discussion}

Paleogeographic events in the divergence of the Pinus phylogeny

Pinus and Picea diverged about 214 Mya at the end of the Triassic (252-201 Mya). Compared with previous studies $[8,9]$, the time between Pinus and Picea was older than the previous result of 174-190 Mya. At the beginning of the Triassic, only one supercontinent Pangea was present

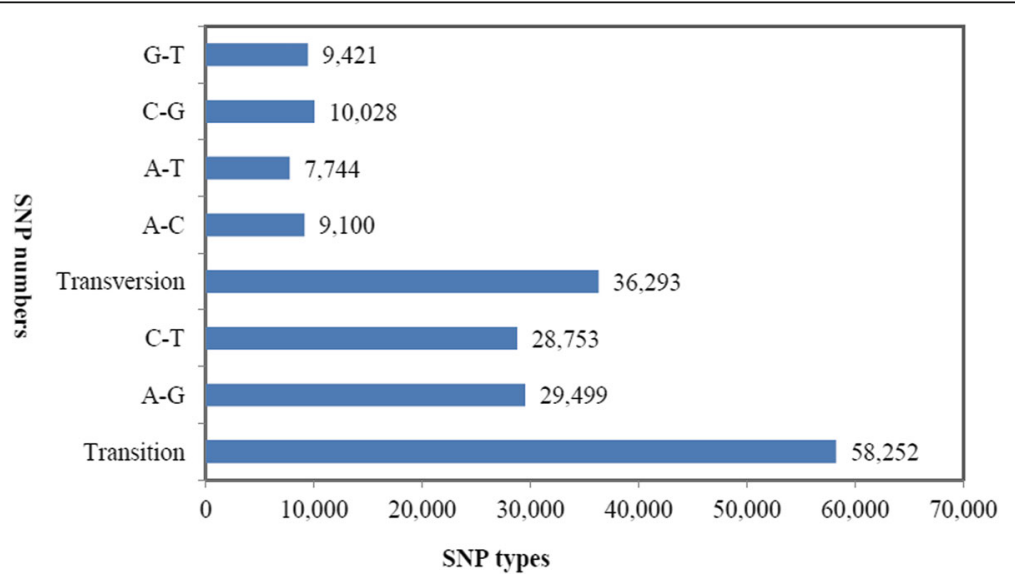

Fig. 3 Distribution of SNPS in the P. kesiya var. langbianensis transcriptome 
Table 3 Transcriptome sequencing in 13 conifer species

\begin{tabular}{|c|c|c|c|c|c|}
\hline Pinus/Picea spp. & Main distribution & Data source & Number of Unigenes & Total length(bp) & Mean length(bp) \\
\hline P. kesiya & $\begin{array}{l}\text { Southwestern China } \\
\text { southeastern Asian }\end{array}$ & Illumina Sequencing & 68,881 & $58,975,225$ & 856 \\
\hline P. tabuliformis & Northern China northern Korea & NCBI SRA(SRR546476) & 10,285 & $8,669,639$ & 843 \\
\hline P. sylvestris & $\begin{array}{l}\text { Middle and high latitudes } \\
\text { of Eurasia }\end{array}$ & PlantGDB & 73,609 & $29,552,311$ & 401 \\
\hline P. pinea & $\begin{array}{l}\text { Mediterranean region } \\
\text { southern Europe }\end{array}$ & NCBI SRA(SRR445497, SRR445498) & 11,403 & $10,122,178$ & 888 \\
\hline P. halepensis & Mediterranean region & NCBI SRA(SRR942848) & 72,028 & $47,224,748$ & 656 \\
\hline P. pinaster & Mediterranean region & PlantGDB & 15,648 & $11,498,176$ & 735 \\
\hline P. contorta & Western North America & PlantGDB & 13,570 & $15,334,600$ & 1130 \\
\hline P. banksiana & North American Canada & PlantGDB & 13,040 & $14,756,117$ & 1132 \\
\hline P. taeda & Southeastern United States & Treegenesdb (v2.01) & 91,046 & $75,408,866$ & 828 \\
\hline P. palustris & Southeastern United States & NCBI SRA(SRR065012) & 15,013 & $14,111,464$ & 940 \\
\hline P. monticola & $\begin{array}{l}\text { Mountains of western } \\
\text { United States and Canada }\end{array}$ & NCBI SRA(SRR1013836) & 86,230 & $33,420,814$ & 388 \\
\hline P. lambertian & $\begin{array}{l}\text { Mountains of western } \\
\text { North America }\end{array}$ & NCBI SRA(SRR064207) & 30,981 & $33,041,789$ & 1067 \\
\hline Picea glauca & $\begin{array}{l}\text { Northern United States } \\
\text { southern/central Canada }\end{array}$ & PlantGDB & 48,619 & $54,962,881$ & 1130 \\
\hline
\end{tabular}

on earth, and the climate was singly warm and dry [24, 25]. The ancient continent was rifting during the late of Triassic with the climate becoming more diverse, during which Pinus and Picea diverged.

The divergence time of the subgenera Strobus and Pinus was about 115 Mya, in the middle of the Cretaceous (145-66 Mya). Compared with previous studies, the time was between the previous results of 85 Mya and 128 Mya $[6,10]$. During the Cretaceous period, the distribution of the land and sea changed rapidly as a result of tectonic activity in the Atlantic Ocean, and large-scale transgression occurred in the coastal areas (a transgressive event) [26]. The species of subgenus Strobus were mainly distributed in the coastal mountains of western North America. In the middle of Cretaceous period, broad shallow seas advanced across central North America. This transgressive event might reflect the geographical speciation of subgenus Strobus and shown to be consistent with the divergence time 115 Mya in our results.

The divergence time of sections Pinus and Trifoliae was about 51 Mya during the Paleogene (66-23 Mya) [27]. There was a dispute over this time in previous studies $[6$, $8-10]$. One suggests the divergence time of section Pinus and section Trifoliae was occurred in the beginning of Paleogene, and another suggests it was in the Late Cretaceous ( 100-66 Ma). Our result supports the former conclusion, but older than the former's result of 44-45 Mya $[8,9]$. The divergence of sections Pinus and Trifoliae may be related to the separation of Laurasia [28] into Eurasia and Laurentia during the Paleocene (66-56 Mya) which is the first epoch of Paleogene.

\section{Salt stress gene selection between coastal and inland Pinus}

Paleogeography (http://www.scotese.com) shows the southern areas of North America had been covered by shallow water during the Paleogene. P. taeda is mainly distributed in the coastal areas of southeastern US, while P. banksiana is an inland Pinus species in the North America and Canada. The regressive event altered the habitat of $P$. taeda from shallow sea into land, which may explain the involvement of the salt stress gene in the selective evolution between $P$. taeda and inland P. banksiana. It might also explain the positive selection of the salt stress gene between coastal P. kesiya var. langbianensis (southwest China) and P. tabuliformis (inland of Northern China).

\section{Abscisic acid stress gene selection between coastal and inland Pinus}

By 50 Mya, the global climate departed from the hot and humid conditions and began a cooling and drying trend towards a series of ice age [29]. Most species of sections Pinus and Trifoliae had gone through this cool stage especially the high-latitude. Production of abscisic acid is increased by cold and drought and acts to help plants withstand these conditions [30-32]. Pinus banksiana occurs in the high-latitude inland regions of North America and Canada, which is possibly related to the positive selection of the abscisic acid gene in this species in comparison to the coastally distributed species $P$. taeda (southeastern US), P. palustris (southeastern US), and P. kesiya var. langbianensis (southwestern China, Southeast Asia). 


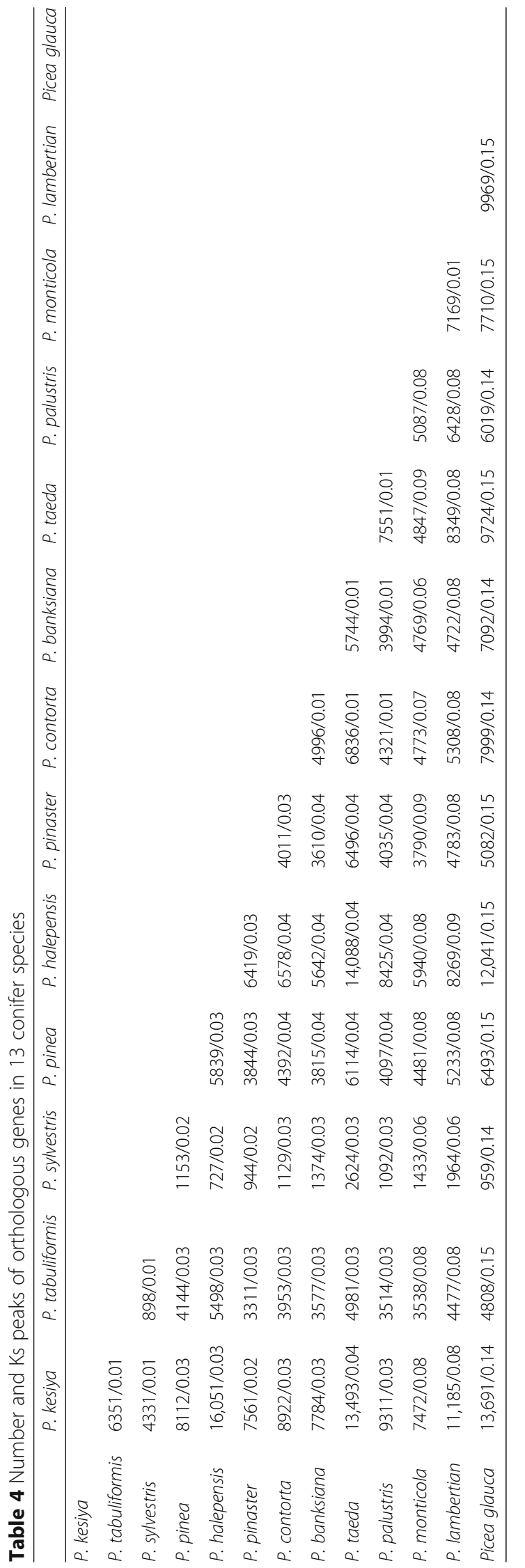




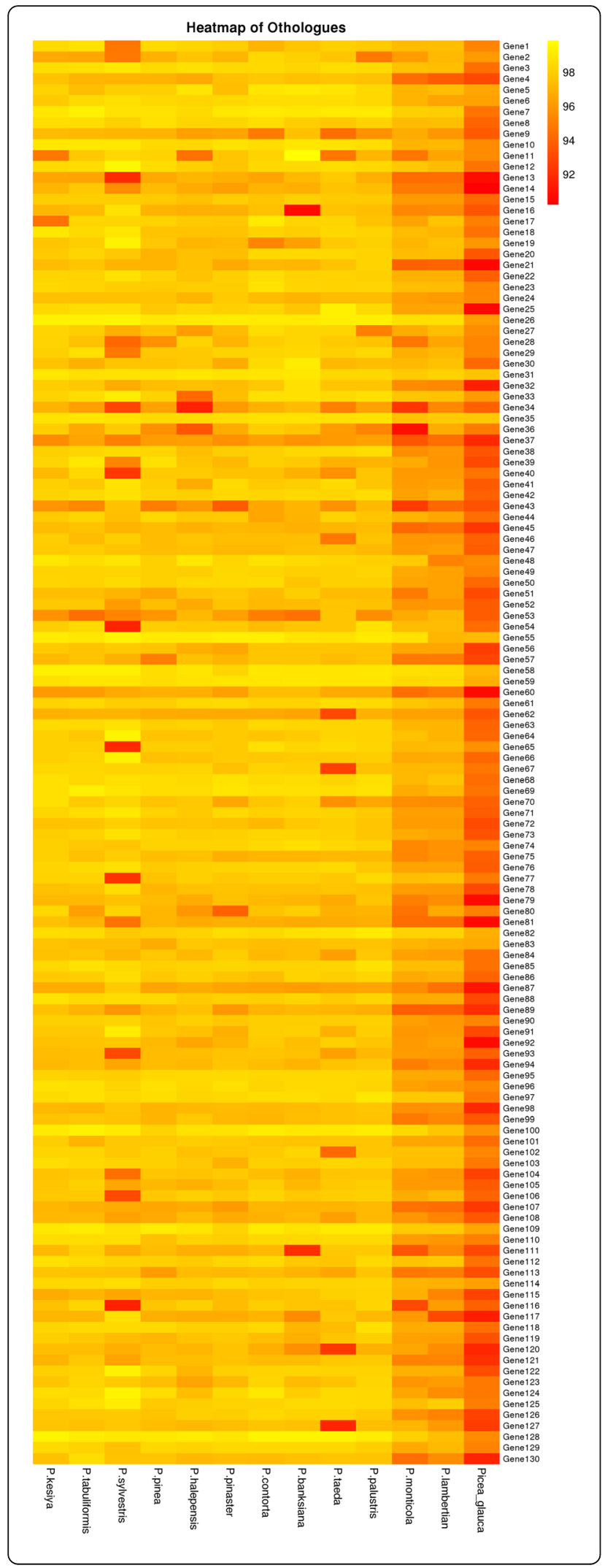

Fig. 4 Functional annotation and divergence between the orthologues of 12 pine and one spruce species. The heat map is based on the 130 putative orthologous transcripts of the 13 species. The orthologues were annotated to different functions based on GO terms (Additional file 3: File S1). Colors indicate similarity from yellow (highly similar) to red (weakly similar)

\section{Conclusions}

In the present study, the phylograms and divergence times were estimated by comparative transcriptomic analysis in 13 conifer species. A number of positive selection genes were found to be associated with environmental factors. The divergence times suggest that plate movement and transgression events caused the geographical speciation which might be the key drives in the divergence of Pinus phylogeny. The analysis of selection evolution suggests salt and abscisic acid-related genes were involved in positive selection between coastal and inland Pinus species. These data are useful for evaluating the different evolutionary patterns between inland and coastal Pinus species. The study shows that tectonic plate movement, and transgression and regression events resulted in changes to the land and sea, and adaptive evolution may have played an important role in the divergence of the Pinus species.

\section{Methods}

\section{RNA extraction and sequencing}

Pine needle tissue, including the cambium, of P. kesiya var. langbianensis was collected from Puer City, Yunnan Province, China. The specimen was identified by Yong-chun $\mathrm{Xu}$ and Juan Wang, whose deposition numbers are 0000651 and 0000652 from the herbarium of Southwest Forestry University. Total RNA was isolated using RNAiso Plus (TaKaRa, Japan). RNA quality was characterized initially on an agarose gel and NanoDrop ND1000 spectrophotometer (NanoDrop Technologies, Wilmington, DE, USA).

Illumina sequencing based on a GAII platform was performed at the Beijing Genomics Institute (Shenzhen, China; http://www.genomics.cn), following the manufacturer's protocols. A fragmentation buffer was added to interrupt the mRNA and thereby generate fragments in the size of range $200 \mathrm{bp}$. The resulting fragments served as a template for the synthesis of the first-strand cDNA, employing random hexamer primers (N6). Second-strand cDNA was synthesized using a SuperScript Double-Stranded cDNA Synthesis kit (Invitrogen, Camarillo, CA), after which it was purified using a QiaQuick PCR extraction kit (Qiagen, Hilden, Germany) and resolved with EB buffer for end repair and poly (A) addition. The products were ligated with one another using sequencing adapters, and a suitable fragment size range was selected for PCR amplification following agarose gel electrophoresis. The resulting library was sequenced using an Illumina HiSeqTM 2000 platform. 


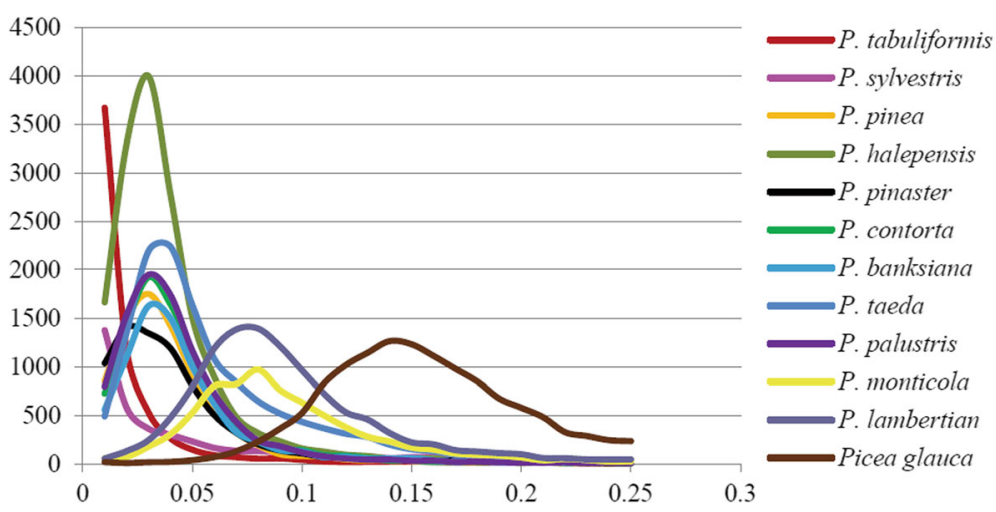

Fig. 5 Distribution of Ks values of the orthologous pairs between $P$. kesiya var. langbianensis and others

\section{Data filtering and de novo assembly}

Image data output from the sequencing device were transformed into raw reads and stored in FASTQ format. These data were filtered to remove raw reads that included adapter sequences or those that were of low quality. Transcriptome assembly was achieved using the short-read assembly program Trinity [33]. The unigenes were divided into either clusters or singletons. BLASTX [34] alignment between each unigene sequence and those lodged in the $\mathrm{Nr}, \mathrm{Nt}$ (Nucleotide database, NCBI), Swiss-Prot, GO (http://www.geneontology.org/), and COG (clusters of orthologous groups) databases were performed, and the best alignments were used to infer the directionality of the unigene. Where the outcomes from the various databases conflicted with one another, the priority order applied was: Nr, Swiss-Prot, and COG. Where no alignment was possible, the software tool ESTScan [35] was used to assign directionality.

\section{Gene annotation and analysis}

Functional annotation was assigned using the protein $(\mathrm{Nr}$ and Swiss-Prot), COG, and GO databases. BLASTX was employed to identify related sequences in the protein databases. The COG database attempts to classify proteins from completely sequenced genomes on the basis of the orthology concept [36]. The aim of GO is to standardize the representation of genes and their products by insisting on a controlled vocabulary and a strictly defined concept [37, 38]. The annotations acquired from $\mathrm{Nr}$ were processed through the Blast2GO program [39] to obtain the relevant GO terms, and these were then analyzed by WEGO software [40] to assign a GO functional classification and to illustrate the distribution of the gene functions.

\section{Identification of SSRs and SNPs}

Unigenes containing putative SSRs and SNPs were identified by MISA and SOAPsnp [41] software. Mono-

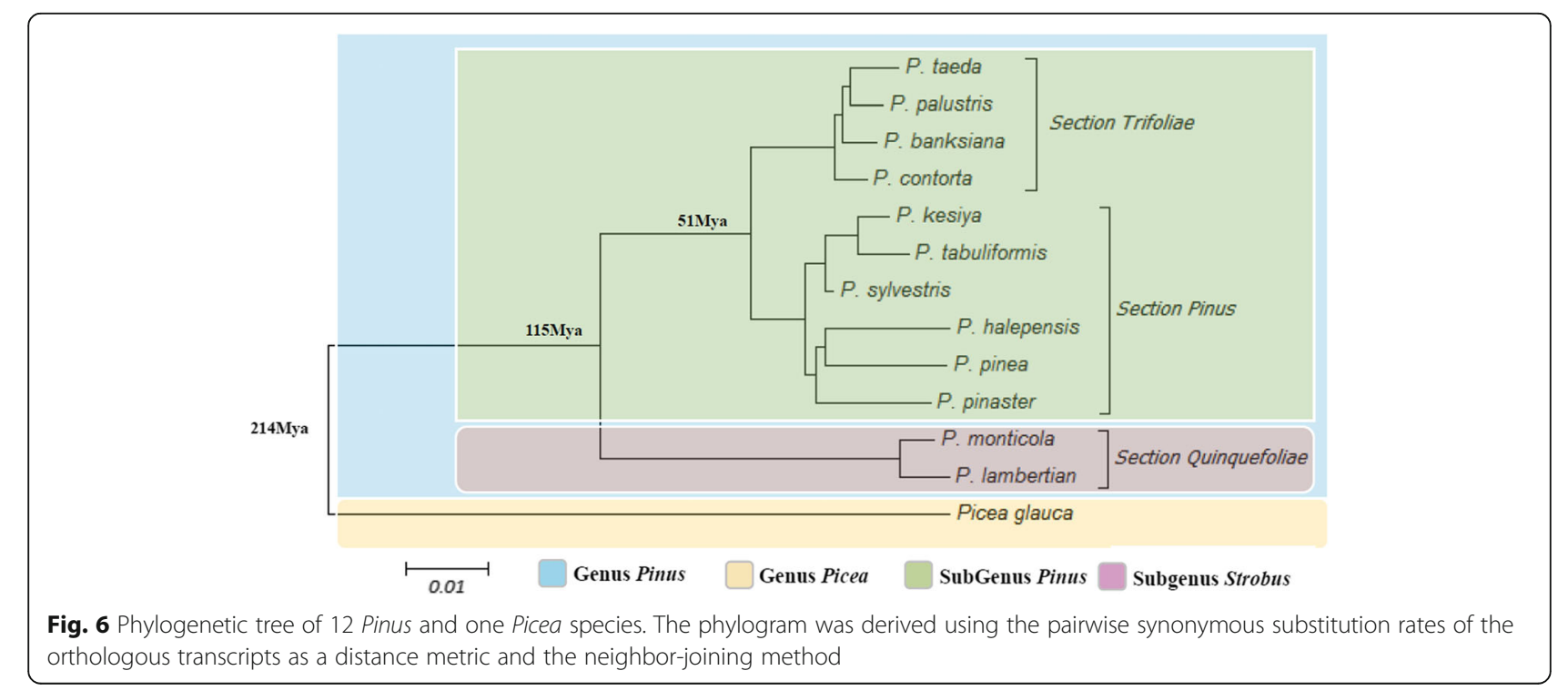




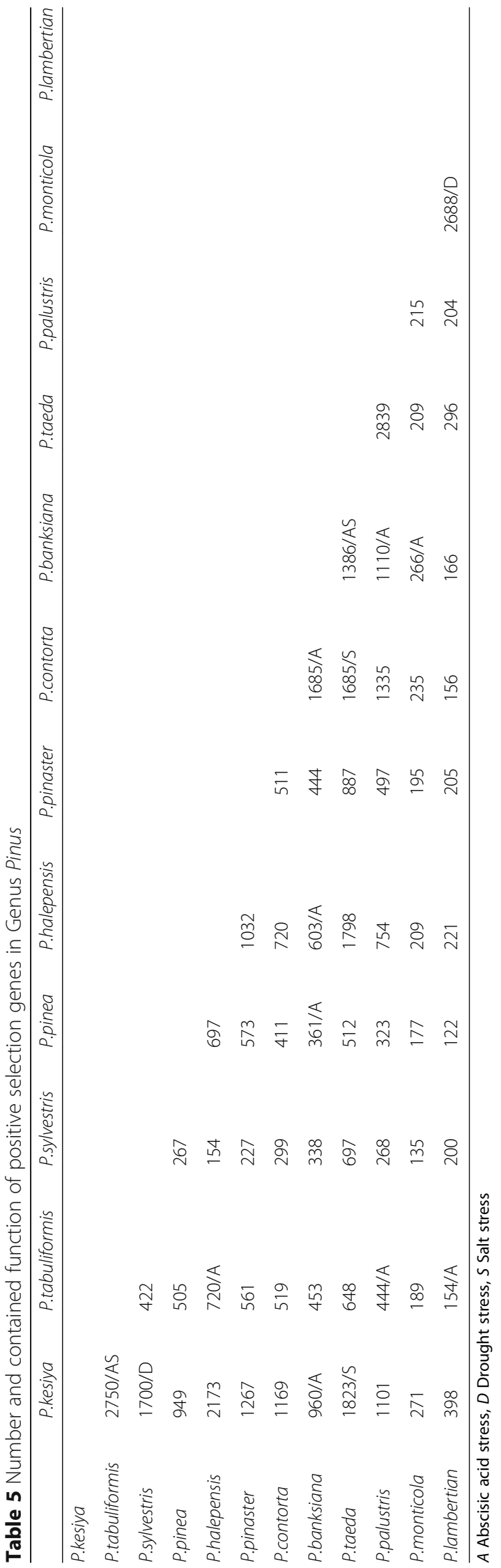


Table $\mathbf{6}$ Information of resistance genes involved in positive selection in Genus Pinus

\begin{tabular}{|c|c|c|c|c|c|c|c|c|}
\hline Funtion & Nr Annotation & Specie1 & Gene1 & Specie2 & Gene2 & $\mathrm{Ka} / \mathrm{Ks}$ & $\mathrm{Ka}$ & Ks \\
\hline Salt stress & NP_001105589.1 & P. tabuliformis & isotig03867 & P. kesiya & CL1082.Contig1_All & $>1$ & 0.0013 & 0 \\
\hline Salt stress & NP_001105589.1 & P. taeda & PITA_000000350-RA & P. banksiana & $\begin{array}{l}\text { PUT-177a-Pinus_ } \\
\text { banksiana-8213 }\end{array}$ & 4.3893 & 0.0873 & 0.0199 \\
\hline Salt stress & NP_001105589.1 & P. taeda & PITA_000000350-RA & P. kesiya & CL1082.Contig1_All & 4.9134 & 0.0994 & 0.0202 \\
\hline Salt stress & NP_001105589.1 & P. taeda & PITA_000000350-RA & P. contorta & $\begin{array}{l}\text { PUT-175a-Pinus_ } \\
\text { contorta-5514 }\end{array}$ & 4.3893 & 0.0873 & 0.0199 \\
\hline Drought stress & AAD37375.1 & P. sylvestris & PUT-187a-Pinus_sylvestris-52,858 & P. kesiya & Unigene23170_All & 1.0047 & 0.0083 & 0.0083 \\
\hline Drought stress & AAD37375.1 & P. lambertian & isotig24007 & P. monticola & comp65821_c3 & $>1$ & 0.0046 & 0 \\
\hline ABA stress & NP_001105719.1 & P. palustris & isotig03955 & P. banksiana & $\begin{array}{l}\text { PUT-177a-Pinus_- } \\
\text { banksiana-10,254 }\end{array}$ & 1.2072 & 0.0054 & 0.0045 \\
\hline ABA stress & NP_001105719.1 & P. kesiya & Unigene11406_All & P. banksiana & $\begin{array}{l}\text { PUT-177a-Pinus_ } \\
\text { banksiana-10,253 }\end{array}$ & 1.166 & 0.0262 & 0.0224 \\
\hline ABA stress & NP_001105719.1 & P. contorta & PUT-175a-Pinus_contorta-11,400 & P. banksiana & $\begin{array}{l}\text { PUT-177a-Pinus_ } \\
\text { banksiana-10,253 }\end{array}$ & 1.2 & 0.0162 & 0.0135 \\
\hline ABA stress & NP_001105719.1 & P. taeda & PITA_000045763-RA & P. banksiana & $\begin{array}{l}\text { PUT-177a-Pinus_ } \\
\text { banksiana-10,254 }\end{array}$ & $>1$ & 0.004 & 0 \\
\hline ABA stress & NP_001105719.1 & P. pinea & isotig05514 & P. banksiana & $\begin{array}{l}\text { PUT-177a-Pinus_ } \\
\text { banksiana-10,253 }\end{array}$ & 1.0145 & 0.05 & 0.0493 \\
\hline ABA stress & NP_001105719.1 & P. halepensis & comp18337_c1 & P. banksiana & $\begin{array}{l}\text { PUT-177a-Pinus_ } \\
\text { banksiana-10,253 }\end{array}$ & 1.2039 & 0.0601 & 0.0499 \\
\hline
\end{tabular}

ABA is Abscisic acid. Sequences showed in Additional file 4: File S2

to hexa-nucleotide SSRs with a minimum repeat unit size of 12 (for mono-), 6 (for di-), 5 (for tri- and tetra-), and 4 (for penta- and hexa-) were identified based on the analysis of the assembled $P$. kesiya unigenes.

\section{Identification of orthologues between the 13 conifer species}

In order to discover the evolutionary patterns of orthologues in Pinus, transcriptome sequences of 11 Pinus and Picea glauca (outgroup) accessions were downloaded from the PlantGDB and NCBI databases (Table 3). Pinus banksiana, P. contorta, P. sylvestris, P. pinaster, and Picea glauca were directly derived from PlantGDB. Pinus taeda was obtained from the NCBI genome database $[42,43]$. Pinus monticola, $P$. lambertiana, P. pinea, P. halepensis, $P$. tabuliformis, and $P$. palustris were obtained from the NCBI SRA database. The 454 RNA dataset was assembled using Newbler (http:// roche-applied-science.com/) software, and the Illumina RNA dataset was assembled by Trinity software. The assembled sequences were combined and clustered with CD-HIT (version 4.0) [44, 45]. Sequences with similarity >95\% were divided into one class, and the longest sequence of each class was treated as a unigene during later processing.

The transcribed sequences were clustered using UCLUST software [46]. Aligned sequences showing 90\% identity were defined as pairs of putative orthologues among the 13 species. The best-hit sequence of each cluster was then used in the subsequent analyses.
Estimation at the synonymous substitution and nonsynonymous substitution levels between orthologues Since unigenes are derived from expressed sequence tag (EST) sequences, have no annotated open reading frames, and may contain frame shift sequencing errors, each member of a pair of sequences was searched using BLASTX against all of the plant protein sequences available in GenBank. The approach used was as described previously [47]. PAML (http://abacus.gene.ucl.ac.uk/software/paml.html) software was used to estimate the non-synonymous substitutions per non-synonymous site (Ka) and the synonymous substitutions per synonymous site (Ks) [48].

\section{Phylogenetic analysis}

As the phylogenetic relationships in Pinus are well understood $[6,7]$, the precise topology is not critical for the purposes of this study. We chose to focus our analyses on the evolutionary patterns and rate of genetic divergence. The synonymous substitutions and non-synonymous substitutions between the orthologues of the 13 conifer species were analyzed as described previously. Phylograms were derived using the pairwise Ks values of the orthologous transcripts as a distance metric based on the neighbor-joining method [49]. Picea glauca was used as an outgroup to root the trees. The distance of two branches was estimated based on the average of all of the pairwise Ks values in two branches of the phylogenetic tree. 


\section{Additional files}

Additional file 1: Figure S1. Distribution of unigene length and depth. (TIFF $7129 \mathrm{~kb}$ )

Additional file 2: Table S1. Gene Ontology (GO) distributions for $P$. kesiya var. langbianensis. Table S2. SSR markers identified in 13 conifer species. Table S3. Gene Ontology (GO) distributions for shared orthologues in 13 conifer species. (XLSX $16 \mathrm{~kb}$ )

Additional file 3: File S1. Go annotation of 130 shared unigenes (PDF $17 \mathrm{~kb}$ )

Additional file 4: File S2. Sequences of resistance unigenes (PDF 14 kb)

\section{Abbreviations}

COG: Clusters of orthologous groups; EST: Expressed sequence tag; GO: Gene Ontology; Ka: Non-synonymous substitutions per non-synonymous site; Ks: Synonymous substitutions per synonymous site; Mya: Million years ago; Nr: NCBI Non-redundant protein database; SNP: Single nucleotide polymorphism; SSR: Simple sequence repeat

\section{Acknowledgements}

We thank LetPub (www.letpub.com) for its linguistic assistance during the preparation of this manuscript. This work was supported by Cloud center of Ali of Southwest Forestry University.

\section{Funding}

This work was supported by Project of National Natural Science Foundation (31660029) and Education Department of Yunnan Province (2015Y287).

\section{Availability of data and materials}

The raw Illumina data obtained in this study were deposited in the NCBI Sequence Read Archive (SRA) under the accession number SRR7778006 and SRR7778007. The deposition numbers of specimen are 0000651 and 0000652 from the herbarium of Southwest Forestry University.

\section{Authors' contributions}

YJZ participated in the sequence alignment and drafted the manuscript. JW participated in the samples preparation and transcriptome sequencing. YC and YJZ participated in the design of the study and performed the statistical analysis. ZX conceived of the study, and participated in its design and coordination and helped to draft the manuscript. All authors read and approved the final manuscript.

\section{Ethics approval and consent to participate}

Not applicable.

\section{Consent for publication}

Not applicable.

\section{Competing interests}

The authors declare that they have no competing interests.

\section{Publisher's Note}

Springer Nature remains neutral with regard to jurisdictional claims in published maps and institutional affiliations.

\footnotetext{
Author details

${ }^{1}$ Key Laboratory for Forest Resources Conservation and Utilization in the Southwest Mountains of China, Ministry of Education, Southwest Forestry University, Kunming 650224, Yunnan, People's Republic of China. ${ }^{2}$ College of Big data and Intelligent Engineering, Southwest Forestry University, Kunming 650224, Yunnan, People's Republic of China. ${ }^{3}$ Eco-development Academy, Southwest Forestry University, Kunming 650224, Yunnan, People's Republic of China. ${ }^{4}$ College of Light industry and Food, Southwest Forestry University, Kunming 650224, Yunnan, People's Republic of China.
}

Received: 12 June 2018 Accepted: 27 September 2018

Published online: 03 October 2018

\section{References}

1. Zanoni TA, Farjon A. World checklist and bibliography of conifers. Brittonia. 1999;51:76. https://doi.org/10.2307/2666560.

2. Richardson DM, Rundel PW. Ecology and biogeography of Pinus: an introduction. Ecol Biogeogr Pinus. 2000; January 1998:3-48.

3. Price RA, Liston A, Strauss SH. 2 phylogeny and systematics of Pinus. In: Ecology and Biogeography of Pinus. 2000. p. 527. https://books.google.com/ books?hl=en\& $\mathrm{r}=\& \mathrm{id}=$ YawYOzQmcHEC\&pgis=1.

4. Ryberg PE, Rothwell GW, Stockey RA, Hilton J, Mapes G, Riding JB. Reconsidering relationships among stem and crown group Pinaceae: oldest record of the genus Pinus from the early cretaceous of Yorkshire, United Kingdom. Int J Plant Sci. 2012;173:917-32. https://doi.org/10.1086/667228.

5. Falcon-Lang HJ, Mages V, Collinson M. The oldest Pinus and its preservation by fire. Geology. 2016;44:303-6.

6. Eckert AJ, Hall BD. Phylogeny, historical biogeography, and patterns of diversification for Pinus (Pinaceae): phylogenetic tests of fossil-based hypotheses. Mol Phylogenet Evol. 2006;40:166-82.

7. Gernandt DS, Geada López G, Ortiz García S, Liston A. Phylogeny and classification of Pinus. Taxon. 2005;54:29-42.

8. Ann W, Syring J, Gernandt DS, Liston A, Cronn R. Fossil calibration of molecular divergence infers a moderate mutation rate and recent radiations for Pinus. Mol Biol Evol. 2007;24:90-101.

9. Niu SH, Li ZX, Yuan HW, Chen XY, Li Y, Li W. Transcriptome characterisation of Pinus tabuliformis and evolution of genes in the Pinus phylogeny. BMC Genomics. 2013;14.

10. Saladin B, Leslie AB, Wüest RO, Litsios G, Conti E, Salamin N, et al. Fossils matter: improved estimates of divergence times in Pinus reveal older diversification. BMC Evol Biol. 2017;17.

11. Fernández-Pozo N, Canales J, Guerrero-Fernández D, Villalobos DP, DíazMoreno SM, Bautista R, et al. EuroPineDB: a high-coverage web database for maritime pine transcriptome. BMC Genomics. 2011;12.

12. Parchman TL, Geist KS, Grahnen JA, Benkman CW, Buerkle CA. Transcriptome sequencing in an ecologically important tree species: assembly, annotation, and marker discovery. BMC Genomics. 2010;11.

13. Wan LC, Zhang H, Lu S, Zhang L, Qiu Z, Zhao Y, et al. Transcriptome-wide identification and characterization of miRNAs from Pinus densata. BMC Genomics. 2012;13.

14. Vendramin E, Dettori MT, Giovinazzi J, Micali S, Quarta R, Verde I. A set of EST-SSRs isolated from peach fruit transcriptome and their transportability across Prunus species: primer note. Mol Ecol Notes. 2007;7:307-10.

15. Wang $H$, Jiang J, Chen S, Qi X, Peng H, Li P, et al. Next-generation sequencing of the Chrysanthemum nankingense (Asteraceae) transcriptome permits large-scale Unigene assembly and SSR marker discovery. PLoS One. 2013;8.

16. Neale DB. Genomics to tree breeding and forest health. Curr Opin Genet Dev. 2007;17:539-44

17. Koenig D, Jimenez-Gomez JM, Kimura S, Fulop D, Chitwood DH, Headland $L R$, et al. Comparative transcriptomics reveals patterns of selection in domesticated and wild tomato. Proc Natl Acad Sci. 2013;110:E2655-62. https://doi.org/10.1073/pnas.1309606110.

18. Davidson RM, Gowda M, Moghe G, Lin H, Vaillancourt B, Shiu SH, et al. Comparative transcriptomics of three Poaceae species reveals patterns of gene expression evolution. Plant J. 2012;71:492-502.

19. Liu T, Tang S, Zhu S, Tang Q, Zheng X. Transcriptome comparison reveals the patterns of selection in domesticated and wild ramie (Boehmeria nivea L. gaud). Plant Mol Biol. 2014:86:85-92.

20. Lu X, Huan L, Gao S, He L, Wang G. NADPH from the oxidative pentose phosphate pathway drives the operation of cyclic electron flow around photosystem I in high-intertidal macroalgae under severe salt stress. Physiol Plant. 2016;156:397-406.

21. Chojak-Koźniewska J, Kuźniak E, Linkiewicz A, Sowa S. Primary carbon metabolism-related changes in cucumber exposed to single and sequential treatments with salt stress and bacterial infection. Plant Physiol Biochem. 2018;123:160-9.

22. Phimchan P, Chanthai S, Bosland PW, Techawongstien S. Enzymatic changes in phenylalanine ammonia-lyase, cinnamic-4-hydroxylase, capsaicin synthase, and peroxidase activities in Capsicum under drought stress. J Agric Food Chem. 2014;62:7057-62. 
23. Sofo A, Scopa A, Nuzzaci M, Vitti A. Ascorbate peroxidase and catalase activities and their genetic regulation in plants subjected to drought and salinity stresses. Int J Mol Sci. 2015;16:13561-78.

24. Harris R, McCall R, Randall O, Bin Tawang MH, Williams R, Fairman JG, et al. Climate change during the Triassic and Jurassic. Geol Today. 2017;33:210-5.

25. Preto N, Kustatscher E, Wignall PB. Triassic climates - state of the art and perspectives. Palaeogeogr Palaeoclimatol Palaeoecol. 2010;290:1-10.

26. Hay WW, RM DC, Wold CN, Wilson KM, Voigt S, Schulz M, et al. Alternative global cretaceous paleogeography. In: Special paper 332: evolution of the cretaceous ocean-climate system; 1999. p. 1-47. https://doi.org/10.1130/08137-2332-9.1.

27. Vandenberghe N, Hilgen FJ, Speijer RP. The Paleogene Period; 2012. https:// doi.org/10.1016/B978-0-444-59425-9.00028-7.

28. Selley R, Robin L, Cocks M, Plimer I. Encyclopedia of geology. Geology. 2005:3345.

29. Zachos J, Pagani H, Sloan L, Thomas E, Billups K. Trends, rhythms, and aberrations in global climate 65 ma to present. Science. 2001;292:686-93.

30. Shinozaki K, Yamaguchi-Shinozaki K, Seki M. Regulatory network of gene expression in the drought and cold stress responses. Curr Opin Plant Biol. 2003;6:410-7.

31. Du H, Wu N, Chang Y, Li X, Xiao J, Xiong L. Carotenoid deficiency impairs ABA and IAA biosynthesis and differentially affects drought and cold tolerance in rice. Plant Mol Biol. 2013;83:475-88.

32. Danquah A, de Zelicourt A, Colcombet J, Hirt H. The role of ABA and MAPK signaling pathways in plant abiotic stress responses. Biotechnol Adv. 2014; 32:40-52.

33. Grabherr MG, Haas BJ, Yassour M, Levin JZ, Thompson DA, Amit I, et al. Fulllength transcriptome assembly from RNA-Seq data without a reference genome. Nat Biotechnol. 2011;29:644-52.

34. Cameron M, Williams HE, Cannane A. Improved gapped alignment in BLAST. IEEE/ACM Trans Comput Biol Bioinforma. 2004;1:116-29.

35. Iseli C, Jongeneel CV, Bucher P. ESTScan: a program for detecting, evaluating, and reconstructing potential coding regions in EST sequences. Proc Int Conf Intell Syst Mol Biol. November 2016;1999:138-48.

36. Tatusov RL. The COG database: a tool for genome-scale analysis of protein functions and evolution. Nucleic Acids Res. 2000;28:33-6. https://doi.org/10. 1093/nar/28.1.33

37. Xia Z, Xu H, Zhai J, Li D, Luo H, He C, et al. RNA-Seq analysis and de novo transcriptome assembly of Hevea brasiliensis. Plant Mol Biol. 2011;77:299-308.

38. Wang Z, Fang B, Chen J, Zhang X, Luo Z, Huang L, et al. De novo assembly and characterization of root transcriptome using Illumina paired-end sequencing and development of cSSR markers in sweetpotato (Ipomoea batatas). BMC Genomics. 2010;11.

39. Conesa A, Götz S, García-Gómez JM, Terol J, Talón M, Robles M. Blast2GO: a universal tool for annotation, visualization and analysis in functional genomics research. Bioinformatics. 2005:21:3674-6.

40. Ye J, Fang L, Zheng H, Zhang Y, Chen J, Zhang Z, et al. WEGO: a web tool for plotting GO annotations. Nucleic Acids Res 2006:34 WEB. SERV. ISS.

41. Li R, Li Y, Fang X, Yang H, Wang J, Kristiansen $K$, et al. SNP detection for massively parallel whole-genome resequencing. Genome Res. 2009:19:1124-32.

42. Neale DB, Wegrzyn JL, Stevens KA, Zimin AV, Puiu D, Crepeau MW, et al. Decoding the massive genome of loblolly pine using haploid DNA and novel assembly strategies. Genome Biol. 2014:15.

43. Zimin A, Stevens KA, Crepeau MW, Holtz-Morris A, Koriabine M, Marçais G, et al. Sequencing and assembly of the 22-Gb loblolly pine genome. Genetics. 2014;196:875-90.

44. Huang Y, Niu B, Gao Y, Fu L, Li W. CD-HIT suite: a web server for clustering and comparing biological sequences. Bioinformatics. 2010;26:680-2.

45. Li W, Godzik A. Cd-hit: a fast program for clustering and comparing large sets of protein or nucleotide sequences. Bioinformatics. 2006;22:1658-9.

46. Edgar RC. Search and clustering orders of magnitude faster than BLAST. Bioinformatics. 2010;26:2460-1.

47. Blanc G. Widespread Paleopolyploidy in model plant species inferred from age distributions of duplicate genes. PLANT CELL ONLINE. 2004;16:1667-78. https://doi.org/10.1105/tpc.021345.

48. Yang Z. PAML 4: phylogenetic analysis by maximum likelihood. Mol Biol Evol. 2007;24:1586-91

49. Tamura K, Dudley J, Nei M, Kumar S. MEGA4: molecular evolutionary genetics analysis (MEGA) software version 4.0. Mol Biol Evol. 2007;24:1596-9.

Ready to submit your research? Choose BMC and benefit from:

- fast, convenient online submission

- thorough peer review by experienced researchers in your field

- rapid publication on acceptance

- support for research data, including large and complex data types

- gold Open Access which fosters wider collaboration and increased citations

- maximum visibility for your research: over $100 \mathrm{M}$ website views per year

At $\mathrm{BMC}$, research is always in progress.

Learn more biomedcentral.com/submissions 